\title{
Effects of task-relevant incentives on the electrophysiological correlates of error processing in major depressive disorder
}

\author{
Avram J. Holmes and Diego A. Pizzagalli \\ Harvard University, Cambridge, Massachusetts
}

\begin{abstract}
Major depressive disorder (MDD) is associated with action-monitoring dysfunction—particularly, disrupted error processing. Whether such dysregulation is further modulated by task incentives is largely unknown. The goal of this study was to investigate possible dysfunctions in error processing in MDD as a function of varying task incentives and clinical profile. To this end, we recorded the error-related negativity (ERN) and error positivity $(\mathrm{Pe})$ in $18 \mathrm{MDD}$ participants and 18 healthy controls during a Stroop task that intermixed no-incentive and reward trials. Relative to controls, MDD participants showed (1) larger ERN irrespective of task incentives, and (2) reduced Pe during reward (but not no-incentive) trials. Moreover, among MDD participants, Pe amplitudes were negatively correlated with depression severity and clinical symptoms. The present findings highlight distinct effects of task incentives on electrophysiological components of error processing and are interpreted within current theories of action monitoring and incentive processing in depression.
\end{abstract}

One aspect of cognition that has attracted considerable interest is the ability to adjust performance with shifting incentives and the consequences of previous events (action monitoring). This capacity allows us to coordinate actions in a manner that maximizes the likelihood of achieving intended goals, even following unexpected changes in the environment. Major depressive disorder (MDD) is characterized by action-monitoring dysfunction - particularly, impaired behavioral performance in situations requiring adaptive strategy shifts (Beats, Sahakian, \& Levy, 1996; Elliott et al., 1996; Holmes \& Pizzagalli, 2008) and decreased approach-related behavior (for a review, see Pizzagalli, Dillon, Bogdan, \& Holmes, in press). Thus, impairments in action monitoring might be partially explained by abnormalities in incentive processing. Providing initial support for this hypothesis, among healthy controls, action monitoring has been found to be modulated by motivational/emotional context and individual differences in reward sensitivity (Boksem, Tops, Kostermans, \& De Cremer, 2008; Holmes \& Pizzagalli, 2007; Luu, Tucker, Derryberry, Reed, \& Poulsen, 2003).

A growing literature suggests that dysregulated activity within structures critically implicated in action monitoring and incentive processing, including the dorsolateral prefrontal cortex (DLPFC) and anterior cingulate cortex (ACC), could contribute to behavioral impairments in MDD (e.g., Alexopoulos et al., 2005; Forbes et al., 2006; Siegle, Thompson, Carter, Steinhauer, \& Thase, 2007). Specifically, dysfunctional ACC activity in MDD has been associated with reduced performance following perceived failure (errors) or negative feedback (Holmes
\& Pizzagalli, 2008; Tucker, Luu, Frishkoff, Quiring, \& Poulsen, 2003), as well as reduced responsiveness to positive reinforcement (e.g., monetary incentives; Forbes et al., 2006). Altogether, prior behavioral and neuroimaging findings raise the possibility that the actionmonitoring system could be differentially modulated by shifts in task-relevant incentives in healthy controls and MDD participants.

Due to the rapid nature of neural processes implicated in action monitoring, research in this area has relied on electrophysiological markers of performance monitoring. Capitalizing on the temporal resolution of event-related potential (ERP) recordings, studies have primarily focused on two response-related waveforms - the error-related negativity (ERN) and error positivity $(\mathrm{Pe})$ - which may represent partially dissociable components of error processing (e.g., Falkenstein, Hoormann, Christ, \& Hohnsbein, 2000; Holroyd \& Coles, 2002; Overbeek, Nieuwenhuis, \& Ridderinkhof, 2005; van Veen \& Carter, 2002).

\section{ERN}

Occurring 50-150 msec following an incorrect response and elicited even when participants are unaware of having committed a mistake (see, e.g., Nieuwenhuis, Ridderinkhof, Blom, Band, \& Kok, 2001; Shalgi, Barkan, \& Deouell, 2009), the ERN is a negative deflection with frontocentral scalp distribution believed to index the automatic detection of events (e.g., errors) that require increased cognitive control (see, e.g., Falkenstein, Hohnsbein, Hoormann, \& Blanke, 1991; Gehring, Goss, Coles, Meyer, \& Donchin, 1993). The function of the ERN re- 
mains under debate, but recent findings suggest that, in addition to automatic error detection, it may reflect a more general estimation of the motivational value of ongoing events (e.g., Boksem et al., 2008; Hajcak, Holroyd, Moser, \& Simons, 2005; Luu, Collins, \& Tucker, 2000).

Consistent with this argument, among healthy controls, ERN amplitude is larger (i.e., more negative) to errors committed when monetary incentives are available (see, e.g., Ganushchak \& Schiller, 2008; Gehring \& Willoughby, 2002; Hajcak, Moser, Yeung, \& Simons, 2005). Along similar lines, in a recent study (Boksem et al., 2008) using an Eriksen flanker task under varying incentive conditions, students high on reward sensitivity showed heightened ERN when failing to earn rewards due to errors, but not when they were punished for committing mistakes. In contrast, participants with high punishment sensitivity showed largest ERN under the punishment condition. Collectively, these findings suggest that the ERN is sensitive to the motivational significance of errors (for a recent review, see Olvet \& Hajcak, 2008).

ERN findings in MDD have been inconsistent. Potentiated ERN has been observed in moderately depressed participants during experimental tasks devoid of incentive manipulations in some (e.g., Alexopoulos et al., 2007; Chiu \& Deldin, 2007; Holmes \& Pizzagalli, 2008) but not all (Ruchsow et al., 2006; Ruchsow et al., 2004) studies, as well as in tasks involving possible penalties (Chiu \& Deldin, 2007), whereas no differences between MDD and control participants emerged within the context of reward (Chiu \& Deldin, 2007). Conversely, in severely depressed samples, reduced ERN amplitudes following errors have emerged under no-incentive conditions, a finding generally attributed to the presence of apathy and/or psychomotor slowing in more severe cases of depression (Schrijvers et al., 2008; Schrijvers, Maas, et al., 2009). Accordingly, under baseline (no-incentive) conditions, MDD participants appear to be characterized by exaggerated automatic responses to perceived failures. However, when errors occur within the context of rewards, group differences may be attenuated due to the fact that healthy controls generate relatively larger ERN during reward conditions than during no-incentive conditions (Ganushchak \& Schiller, 2008; Gehring \& Willoughby, 2002; Hajcak, Moser, et al., 2005), whereas MDD participants may lack this potentiation due to anhedonia and blunted reward responsiveness (e.g., Forbes et al., 2006; Pizzagalli, Holmes, et al., 2009; Pizzagalli, Iosifescu, Hallett, Ratner, \& Fava, 2009). On the basis of these arguments and prior studies (Chiu \& Deldin, 2007; Holmes \& Pizzagalli, 2008), we hypothesized that, relative to controls, MDD participants would show larger ERN to errors, particularly under no-incentive conditions.

\section{Pe}

The Pe is a positive voltage deflection peaking approximately 200-400 msec after error commission (Falkenstein et al., 2000). Although the functional significance of the Pe remains to be delineated, it has been hypothesized to reflect error awareness, affective appraisal of errors, or processes implicated in posterror behavioral adjustments (e.g., Hajcak, McDonald, \& Simons, 2003; Nieuwenhuis et al., 2001;
Shalgi et al., 2009; for reviews, see Falkenstein et al., 2000; Overbeek et al., 2005). In addition, similarities between the $\mathrm{Pe}$ and $\mathrm{P} 3 \mathrm{~b}$ components have led to the hypothesis that the Pe might be linked to the motivational significance of errors (Overbeek et al., 2005). In line with this argument, Pe amplitude has been found to be positively correlated with individual differences in reward sensitivity (e.g., Boksem et al., 2008; Boksem, Tops, Wester, Meijman, \& Lorist, 2006) and larger when errors are salient (e.g., Leuthold \& Sommer, 1999). These findings have been interpreted as suggesting that, following a mistake in performance, the Pe might reflect a mechanism to prevent the occurrence of subsequent errors, maximizing future rewards (i.e., approach motivation/reward seeking; Boksem et al., 2006).

Pe findings in depression have been equally inconsistent. Specifically, whereas reduced Pe has been described in severely depressed individuals (Alexopoulos et al., 2007; Schrijvers et al., 2008; Schrijvers, de Bruijn, et al., 2009), such findings in moderately depressed participants have not emerged (Chiu \& Deldin, 2007; Compton et al., 2008; Holmes \& Pizzagalli, 2008). Prior null findings in participants with moderate levels of depressive symptoms were interpreted as suggesting that disruption in later stages of error processing might emerge only in samples of treatment-resistant patients and/or geriatric patients with depression (Holmes \& Pizzagalli, 2008). However, it is important to emphasize that null findings have been observed either in experimental tasks without manipulations of incentive motivation (Compton et al., 2008; Holmes \& Pizzagalli, 2008) or in tasks involving separate blocks of reward and no-incentive trials (Chiu \& Deldin, 2007). Because reduced Pe in severe depression has been attributed to anhedonia and apathy (Schrijvers, de Bruijn, et al., 2009), and in light of findings in healthy controls implicating the Pe in processing the motivational significance of errors (Overbeek et al., 2005), we reasoned that Pe differences might emerge in tasks manipulating incentive motivation on a trial-by-trial basis. On the basis of these arguments and on findings highlighting reduced reward responsiveness in depression (e.g., Pizzagalli, Holmes, et al., 2009; Pizzagalli, Iosifescu, et al., 2009), we hypothesized that moderately depressed participants would display reduced $\mathrm{Pe}$, particularly within the context of reward trials.

In sum, the goal of the present study was to investigate putative dysfunctions in error processing in MDD as a function of varying task incentives and clinical profile. To this end, 128-channel ERPs were recorded as healthy controls and unmedicated, moderately depressed MDD participants performed a Stroop task under different incentive conditions (no incentive vs. reward). In addition to testing hypotheses concerning group differences in ERN and Pe as a function of task incentives, we performed correlational analyses to investigate whether these error-related ERP components were further modulated by depression severity and anhedonia. In light of inconsistent findings in the literature (e.g., Alexopoulos et al., 2007; Holmes \& Pizzagalli, 2008; Schrijvers, Maas, et al., 2009), these latter analyses were considered exploratory. 


\section{METHOD}

\section{Participants}

Eighteen participants with MDD and 18 controls were recruited from the community through flyers and Internet postings. General inclusion criteria included right-handedness (Chapman \& Chapman, 1987), age between 18 and 55 years, and normal or corrected-tonormal vision. Participants were excluded if they reported neurological disorders; a history of electroconvulsive therapy, seizures, or head injury resulting in loss of consciousness; or past/current substance abuse or dependence (with the exception of alcohol and marijuana abuse $>1$ year ago). None of the participants had had prior research experience in our laboratory; consequently, the present sample has not been included in any prior publications (Holmes \& Pizzagalli, 2008).

Depressed participants met Diagnostic and Statistical Manual of Mental Disorders (4th ed.) (DSM-IV; American Psychiatric Association, 1994) diagnostic criteria for MDD, as assessed by the Structured Clinical Interview for the DSM-IV (SCID; First, Spitzer, Gibbon, \& Williams, 2007). MDD participants were excluded if they reported current or past psychotic symptomatology or met criteria for any current Axis I diagnosis other than MDD (with the exception of simple phobia, $n=2$ ) and/or if they had received psychotropic medication within the previous 2 weeks ( 4 weeks for neuroleptics and benzodiazepines, 6 weeks for fluoxetine, and 6 months for dopaminergic drugs). Healthy controls were excluded if they reported current or past psychopathology (as assessed by the SCID) or psychological treatment. Three participants performed at or worse than chance levels in the behavioral task, and their data were excluded from the analyses (MDD, $n=2$; controls, $n=1$ ).

To investigate possible relationships between ERN/Pe amplitudes and clinical symptoms, the Beck Depression Inventory-II (BDI-II; Beck, Steer, \& Brown, 1996) and Mood and Anxiety Symptom Questionnaire (MASQ; Watson et al., 1995) were administered. The MASQ assesses anxiety-specific symptoms (anxious arousal, AA), depression-specific symptoms (anhedonic depression, AD), and general distress symptoms (general distress-anxious symptoms, GDA; general distress-depressive symptoms, GDD).

\section{Procedure}

The study consisted of two separate sessions. In the first session, written informed consent was collected after the study was described. Subsequently, a SCID was administered by a masters-level interviewer to confirm study eligibility. To evaluate diagnostic reliability, audiotapes from 18 SCID interviews were randomly selected and independently rated by a second interviewer $(\kappa=1)$. Within 1 week of establishing study eligibility, we invited the participants to the second session, during which they completed self-report questionnaires (BDI, MASQ) and performed a modified version of the Stroop task while 128-channel EEG data were collected. After the task, participants were fully debriefed and received $\$ 32$ for their participation. The Committee on the Use of Human Subjects at Harvard University approved the protocol.

\section{Apparatus}

We collected 128-channel EEG data within an acoustically and electrically shielded room using the Geodesic Sensor Net system (Electrical Geodesics, Inc., Eugene, OR). Data were collected using a $250-\mathrm{Hz}$ sampling rate (bandwidth, $0.01-100 \mathrm{~Hz}$ ) and the vertex electrode $(\mathrm{Cz})$ as the recording reference. Impedances were kept below $50 \mathrm{~K} \Omega$. E-Prime software (Psychology Software Tools, Inc., Pittsburgh, PA) was used to present the experiment and to interface with the EEG NetStation acquisition software.

\section{Task}

All participants completed a modified version of the Stroop task. The stimuli consisted of three words (RED, GREEN, and BLUE) printed in one of three ink colors (red, green, and blue). Each probe could be either congruent (e.g., the word BLUE printed in blue ink) or incongruent (e.g., the word RED printed in green ink). Responses were made using the index, middle, and ring fingers of the right hand. Participants were instructed to press whichever of the buttons, identified by colored stickers, corresponded to the ink color of each stimulus as quickly and as accurately as possible.

Prior to the task, participants completed two 24-trial practice blocks. Reaction times (RTs) from the second practice block were used to calculate individual windows for early and late responses (see below). During practice, each trial began with the presentation of a fixation cross $(250 \mathrm{msec})$, followed by a Stroop probe $(150 \mathrm{msec})$ and a jittered intertrial interval (ITI; $1,850-1,950 \mathrm{msec}$ ). Upon completing the practice, participants were informed that, on the basis of their task performance, they would have the opportunity to earn $\$ 20$ in addition to their base reimbursement $(\$ 10 / \mathrm{h})$. Unlike a prior study that featured separate blocks with and without incentives (Chiu \& Deldin, 2007), the present design incorporated trial-to-trial shifts in task-relevant incentives, allowing us to compare reward and noincentive conditions while minimizing potential confounds inherent in block designs (e.g., habituation, anticipation, set/strategy effects). Accordingly, participants were informed that a visual cue signaling either the possibility of a rewarding outcome $(+\$)$ or no monetary incentive $(0 \$)$ would be presented at the beginning of each trial. Participants were also told that, in order to maximize their earnings, they should respond as quickly and accurately as possible and that each trial would end with feedback informing them about the outcome.

The behavioral task consisted of six blocks, each involving 100 trials. In each block, $50 \%$ of the trials were reward and no-incentive trials, respectively. Within each block, $36 \%$ of the stimuli were incongruent (64 congruent and 36 incongruent trials/block), evenly distributed across the reward/no-incentive trials. Stimuli were presented using a pseudorandomized sequence in which no more than three repetitions of incongruent/congruent trials, a given word/color, or a reward/no-incentive cue type were allowed. Thus, each trial involved a cue manipulation and task-relevant feedback. Experimental trials began with the presentation of a cue $(1,250 \mathrm{msec})$, which was followed by a jittered interstimulus interval (ISI; 950-1,050 msec), a Stroop probe $(150 \mathrm{msec})$, a second jittered ISI $(1,150-1,250 \mathrm{msec})$, the feedback (200 msec), and a variable ITI (950-1,500 msec).

For each trial, correct responses made within the individually calculated response window-that is, $85 \%$ of each participant's mean RT from the second practice block-were followed by positive feedback (a schematic smiling face). If participants responded incorrectly (or correctly, but outside the response window), they were presented with negative feedback (a schematic frowning face). Participants were informed that, for a reward-cue trial, positive feedback indicated that they had successfully earned money for that trial, whereas negative feedback meant that they had failed to earn additional money. For the no-incentive trials, participants were informed that neither the positive nor the negative feedback would affect their earnings. Participants did not receive feedback about their cumulative earnings during the task. To account for potential performance drifts over time, the response window was recalculated at the middle and end of each behavioral block.

\section{Data Reduction}

Behavioral data. As noted above, participants who performed at chance or worse than chance levels were excluded from the analyses (MDD, $n=2$; control, $n=1)$. For the remaining $\operatorname{MDD}(n=16)$ and control $(n=17)$ participants, trials without a response or with RTs lower than $150 \mathrm{msec}$ or higher than $1,000 \mathrm{msec}$ were excluded. Additionally, to reduce the potential effect of outliers, trials with RTs beyond the mean \pm 3 SDs (following $\ln$ transformation) were excluded from all analyses [total number of excluded trials: MDD, $M=1.63, S D=3.10$; control, $0.77 \pm 1.20 ; t(31)=1.04, p=$ .31].

ERP data. Data were analyzed in BrainVision (Brain Products $\mathrm{GmbH}$, Gilching, Germany). First, an independent component analysis was utilized to remove activity associated with blinks, eye movements, and other artifacts (Jung et al., 2000). Channels with corrupted 
signals were replaced individually through spatially weighted linear interpolations. Semiautomatic artifact detection was performed to identify remaining artifacts (maximal amplitude, $\pm 75 \mu \mathrm{V}$; withinsegment absolute amplitude difference, $150 \mu \mathrm{V}$; gradients, $50 \mu \mathrm{V}$ ). The ERP analyses focused on the ERN, its complement for correct responses [correct-response negativity $(\mathrm{CRN})]$, and the Pe. Responselocked ERPs were computed $200 \mathrm{msec}$ prior to and $924 \mathrm{msec}$ following a response. Mirroring the behavioral data analyses, ERPs were computed only for trials in which participants made a response. Data were then band-pass filtered ( $0-30 \mathrm{~Hz}, 12 \mathrm{~dB}$ /octave), baseline corrected ( -200 to $-100 \mathrm{msec}$ pre-response onset), and rederived to an average reference. Grand mean ERP waveforms were calculated by averaging data across conditions and groups.

ERN/CRN. Amplitudes for the ERN and CRN were calculated separately following responses for the reward and no-incentive trials at midline electrode sites $(\mathrm{Fz}, \mathrm{FCz}$, and $\mathrm{Cz})$, where the $\mathrm{ERN} /$ CRN are largest (e.g., Falkenstein et al., 1991; Gehring et al., 1993; Holmes \& Pizzagalli, 2008). Following prior studies, and to minimize the effects of possible slow-wave components, ERN/CRN amplitudes were calculated as the difference between the most negative peak within the time window $0-150 \mathrm{msec}$ after incorrect and correct responses and the preceding positive peak $-80 \mathrm{msec}$ prior to and $80 \mathrm{msec}$ following the response (e.g., Schrijvers et al., 2008; Ullsperger \& von Cramon, 2006).

Pe. For each participant and condition (reward, no incentive), the amplitude of the Pe following incorrect responses was defined as the most positive peak $170-500 \mathrm{msec}$ following an incorrect response at electrodes CPz and Pz (see, e.g., Schrijvers et al., 2008; Ullsperger $\&$ von Cramon, 2006).

The cortical regions implicated in adaptive responding to errors partially overlap with those associated with response conflict (Ullsperger \& von Cramon, 2001). To avoid confounding these effects, ERN/CRN and Pe waveforms are traditionally scored only for incongruent trial errors or while errors in incongruent and congruent trials are simultaneously considered (e.g., Holmes \& Pizzagalli, 2008; Schrijvers et al., 2008). However, due to the limited number of errors in the congruent condition, condition (reward, no incentive) $\times$ trial type (congruent, incongruent) analyses were not possible. Accordingly, analyses examined group differences in ERN/CRN and Pe amplitudes/latencies during the reward and no-incentive conditions (i.e., collapsing across incongruent and congruent trials). Three MDD participants were lost due to insufficient numbers of artifact-free error responses ( $\leq 10$ errors per condition). The remaining MDD $(n=13)$ and control $(n=17)$ participants did not differ with respect to demographic variables $(p s>.21)$ or to the number of available segments [reward error, $25.62 \pm 18.09$ vs. $25.41 \pm 11.68$; no-incentive error, $27.23 \pm 22.21$ vs. $27.18 \pm 12.06 ; t \mathrm{~s}(28)<0.037$, $p \mathrm{~s}>$.97].

\section{Statistical Analyses}

Exploratory analyses revealed no significant effects of gender or ethnicity; therefore, these variables were not further considered.

Behavioral data. Behavioral analyses focused on group differences in overall RT and accuracy, as well as incongruency (Stroop) effects. Increased Stroop effect scores $\left(\mathrm{RT}_{\text {incongruent }}-\mathrm{RT}_{\text {congruent }}\right)$ and ( accuracy $_{\text {congruent }}-$ accuracy $_{\text {incongruent }}$ ) are indicative of impairments in cognitive control. For accuracy and RT scores, separate mixed $2 \times 2 \times 2$ ANOVAs with group (MDD participants, controls) as the between-subjects factor and trial type (incongruent, congruent) and condition (reward, no incentive) as repeated measures were conducted. Due to the inclusion of incentive cues and task-relevant feedback, as well as relatively long delay between an error and a subsequent response $(\sim 4 \mathrm{sec})$, posterror and postconflict behaviors were not examined.

ERN. Preliminary ANOVAs revealed no ERN, CRN, or Pe latency differences across groups or conditions. Thus, the ANOVAs focused on amplitudes. For the ERN/CRN analyses, a mixed $2 \times$ $2 \times 2 \times 3$ ANOVA with group (MDD participants, controls), condition (reward, no incentive), response (correct, incorrect) and site
Table 1

Summary of Demographic and Self-Report Data

\begin{tabular}{|c|c|c|c|c|}
\hline \multirow[b]{2}{*}{ Variable } & \multicolumn{2}{|c|}{$\begin{array}{c}\text { MDD } \\
\text { Participants }\end{array}$} & \multicolumn{2}{|c|}{$\begin{array}{c}\text { Control } \\
\text { Participants }\end{array}$} \\
\hline & $M$ & $S D$ & $M$ & $S D$ \\
\hline Age* $^{*}$ & 36.44 & 13.06 & 34.82 & 9.39 \\
\hline Years of education* & 16.06 & 2.41 & 15.47 & 1.88 \\
\hline Percent female* & 68.8 & $\mathrm{n} / \mathrm{a}$ & 47.1 & $\mathrm{n} / \mathrm{a}$ \\
\hline Percent Caucasian* & 87.5 & $\mathrm{n} / \mathrm{a}$ & 82.4 & $\mathrm{n} / \mathrm{a}$ \\
\hline BDI-II ${ }^{* * *}$ & 26.75 & 9.07 & 1.00 & 1.12 \\
\hline $\mathrm{AA}^{* *}$ & 21.44 & 4.63 & 17.94 & 1.20 \\
\hline $\mathrm{AD}^{* * *}$ & 86.69 & 10.44 & 48.91 & 7.76 \\
\hline GDA $^{* * *}$ & 20.44 & 5.25 & 12.88 & 1.87 \\
\hline $\mathrm{GDD}^{* * *}$ & 37.88 & 10.14 & 13.49 & 1.68 \\
\hline
\end{tabular}

Note-MDD, major depressive disorder; BDI-II, Beck Depression Inventory-II; AA, anxious arousal symptoms; $\mathrm{AD}$, anhedonic depression symptoms; GDA, general distress-anxious symptoms; GDD, general distress-depressive symptoms. ${ }^{*}$ n.s. ${ }^{* *} p<.005 .{ }^{* * *} p<.001$.

( $\mathrm{Fz}, \mathrm{FCz}, \mathrm{Cz}$ ) as factors was run (i.e., collapsing across responses for incongruent and congruent stimuli). When applicable, the Greenhouse-Geisser correction was applied (adjusted $p$ and $\varepsilon$ values are reported).

Pe. For the Pe, a $2 \times 2 \times 2$ ANOVA with group (MDD participants, controls), condition (reward, no incentive), and site $(\mathrm{CPz}, \mathrm{Pz})$ was performed. Effect sizes are reported in the form of $\eta_{\mathrm{p}}^{2}$ values. Post hoc Newman-Keuls tests were performed in the event of significant ANOVA findings. For the sake of brevity, only ERP effects involving group and condition are presented in detail. Other findings are available upon request from the authors via e-mail (dap@ wjh.harvard.edu).

\section{RESULTS}

\section{Demographic and Self-Report Data}

The demographic and self-report data are summarized in Table 1. The MDD and control groups did not differ with respect to gender $\left[\chi^{2}(1)=1.56, p=.21\right]$, age $[t(31)=0.41, p=.69]$, ethnicity $\left[\chi^{2}(1)=0.17, p=.68\right]$, or education $[t(31)=0.79, p=.44]$. As expected, the unmedicated MDD participants reported significantly higher depressive symptoms $[t(31)=11.28, p<.001]$, with BDI scores in the moderate range $(M=26.75, S D=$ 9.07). MDD participants also reported significantly higher

Table 2

Summary of Accuracy and Reaction Time During the Stroop Task

\begin{tabular}{|c|c|c|c|c|}
\hline \multicolumn{2}{|c|}{$\begin{array}{c}\text { MDD } \\
\text { Participants }\end{array}$} & \multicolumn{2}{|c|}{$\begin{array}{c}\text { Control } \\
\text { Participants }\end{array}$} & \multirow[b]{2}{*}{$p$ Value } \\
\hline$M$ & $S D$ & $M$ & $S D$ & \\
\hline \multicolumn{5}{|c|}{ Accuracy } \\
\hline .88 & .11 & .85 & .07 & .34 \\
\hline .94 & .06 & .94 & .03 & .86 \\
\hline .89 & .09 & .87 & .08 & .34 \\
\hline .94 & .05 & .94 & .03 & .87 \\
\hline
\end{tabular}

$\begin{array}{llllll}\text { Incongruent no-incentive } & .88 & .11 & .85 & .07 & .34 \\ \text { Congruent no-incentive } & .94 & .06 & .94 & .03 & .86 \\ \text { Incongruent reward } & .89 & .09 & .87 & .08 & .34 \\ \text { Congruent reward } & .94 & .05 & .94 & .03 & .87\end{array}$

Congruent reward

Reaction Time

$\begin{array}{llllll}\text { Incongruent no-incentive } & 532.80 & 109.00 & 497.34 & 86.20 & .31\end{array}$

$\begin{array}{lllllll}\text { Congruent no-incentive } & 467.71 & 65.45 & 445.02 & 67.87 & .34\end{array}$

$\begin{array}{lllllll}\text { Incongruent reward } & 517.16 & 109.25 & 489.02 & 92.46 & .43\end{array}$

$\begin{array}{llllll}\text { Congruent reward } & 451.06 & 62.99 & 434.31 & 62.35 & .45\end{array}$

Note-Major depressive disorder (MDD) participants, $n=16$; control participants, $n=17$. 
Table 3

Summary of Significant ANOVA Effects for ERN, CRN, and Pe Amplitudes

\begin{tabular}{lrrrr}
\hline \multicolumn{1}{c}{ Contrast } & \multicolumn{1}{c}{$F$} & $d f$ & $p$ Value & \multicolumn{1}{c}{$\eta_{\mathrm{p}}^{2}$} \\
\hline ERN/CRN & & & & \\
$\quad$ Site & 29.57 & 2,56 & $<.001$ & .51 \\
Response & 72.17 & 1,28 & $<.001$ & .72 \\
Group & 3.07 & 1,18 & .091 & .10 \\
Group $\times$ response & 4.22 & 1,28 & .049 & .13 \\
Pe $^{\text {b }}$ & & & & \\
Site & 17.85 & 1,28 & $<.001$ & .39 \\
$\quad$ Group $\times$ condition & 4.52 & 1,28 & .043 & .14
\end{tabular}

Note-ERN, error-related negativity; CRN, correct-response negativity; Pe, error positivity. ${ }^{a}$ Effects emerging from group $\times$ condition (no incentive, reward) $\times$ response (correct, incorrect) $\times$ site ANOVAs (collapsed across incongruent and congruent trials). beffects emerging from group $\times$ condition (no incentive, reward) $\times$ site ANOVAs (collapsed across incongruent and congruent trials).

Table 4

Mean (and $S D$ ) ERN, CRN, and Pe Amplitudes for MDD and Control Participants (Averaged Across Electrodes of Interest)

\begin{tabular}{|c|c|c|c|c|c|}
\hline & \multicolumn{2}{|c|}{$\begin{array}{c}\text { MDD } \\
\text { Participants }\end{array}$} & \multicolumn{2}{|c|}{$\begin{array}{c}\text { Control } \\
\text { Participants }\end{array}$} & \multirow[b]{2}{*}{$p$ Value } \\
\hline & $M$ & $S D$ & $M$ & $S D$ & \\
\hline \multicolumn{6}{|l|}{ ERN/CRNa } \\
\hline ERN & -4.65 & 2.86 & -2.94 & 1.77 & .053 \\
\hline $\mathrm{CRN}$ & -1.04 & 0.13 & -0.74 & 0.09 & n.s. \\
\hline \multicolumn{6}{|l|}{$\mathrm{Peb}$} \\
\hline No incentive & 2.75 & 3.76 & 3.23 & 3.47 & n.s. \\
\hline Reward & 2.07 & 3.65 & 4.37 & 4.47 & $<.004$ \\
\hline
\end{tabular}

Note-ERN, error-related negativity; CRN, correct-response negativity; Pe, error positivity; MDD, major depressive disorder. ${ }^{a}$ Averaged across reward and no-incentive feedback, as well as incongruent and congruent trials (MDD participants, $n=13$; control participants, $n=17$ ). $\quad{ }^{\mathrm{b}}$ Averaged across incongruent and congruent trials (MDD participants, $n=$ 13; control participants, $n=17$ ).

scores on each of the four MASQ subscales [all $t \mathrm{~s}(31)>$ 2.93, all $p s<.005]$.

\section{Behavioral Data}

Table 2 summarizes accuracy and RT data across the various conditions for the two groups. For accuracy, the main effect of trial type was significant $[F(1,31)=44.51$, $\left.p<.001 ; \eta_{\mathrm{p}}^{2}=.60\right]$, due to the expected higher accuracy for congruent $(.94 \pm .042)$ than for incongruent $(.87 \pm .085)$ trials. For RTs, the main effect of trial type was also significant $\left[F(1,31)=47.80, p<.001 ; \eta_{\mathrm{p}}^{2}=.61\right]$, due to the expected slower RTs for incongruent (509.08 $\pm 98.60 \mathrm{msec})$ than for congruent $(449.53 \pm 83.86 \mathrm{msec})$ trials. Additionally, a main effect of condition emerged $[F(1,31)=$ 20.70, $p<.001 ; \eta_{\mathrm{p}}^{2}=.40$ ], driven by quicker RTs for reward $(472.89 \pm 79.32 \mathrm{msec})$ than for no-incentive $(485.72 \pm 80.24 \mathrm{msec})$ trials, indicating that our reward manipulation was successful. Contrary to our hypotheses, no effects involving group emerged for either accuracy or RT data (all $F \mathrm{~s}<2.43$, all $p \mathrm{~s}>.13$ ).

\section{ERN/CRN}

ERN/CRN analysis included no-incentive versus reward trials collapsed across incongruent and congruent trials.
Tables 3 and 4 summarize significant effects emerging from the group $\times$ condition $\times$ response $\times$ site ANOVA on the ERN and CRN amplitudes. The main effect of site was significant $\left[F(2,56)=29.57, p<.001 ; \eta_{\mathrm{p}}^{2}=.51\right]$, driven by more negative waveforms at $\mathrm{Fz}(-2.95 \pm 1.58 \mu \mathrm{V})$ and $\mathrm{FCz}(-2.59 \pm 1.83 \mu \mathrm{V})$ than at $\mathrm{Cz}(-1.47 \pm 1.78 \mu \mathrm{V}$; both $p \mathrm{~s}<.001)$, as well as at $\mathrm{Fz}$ than at $\mathrm{FCz}(p<.04)$. As expected, the main effect of response was significant $\left[F(1,28)=72.17, p<.001 ; \eta_{\mathrm{p}}^{2}=.72\right]$, due to more negative amplitudes for the ERN $(-3.79 \pm 2.42 \mu \mathrm{V})$ than for the CRN $(-0.89 \pm 0.11 \mu \mathrm{V})$. Of primary importance, a significant group $\times$ response interaction emerged $\left[F(1,28)=4.22, p=.049 ; \eta_{\mathrm{p}}^{2}=.13\right]$, indicating that groups differed significantly in their relative potentiation to error responses. The main effect of group was only trending $\left[F(1,28)=3.07, p=.091 ; \eta_{\mathrm{p}}^{2}=.10\right]$. Follow-up

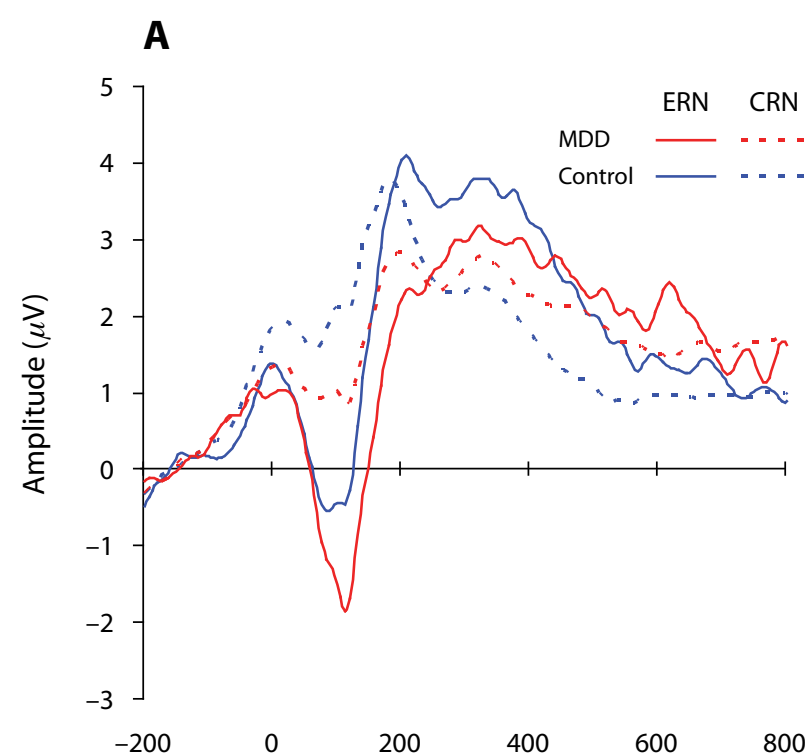

Time (msec)

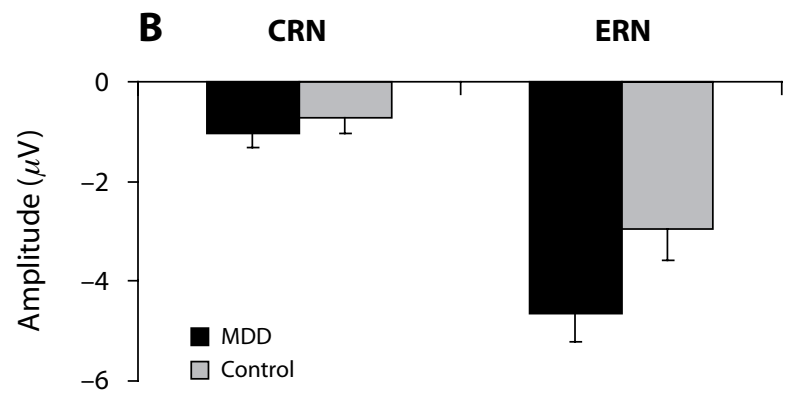

Figure 1. (A) Response-locked waveforms for major depressive disorder (MDD; $n=13)$ and control $(n=17)$ participants. To reflect the significant group $\times$ response interaction and to highlight the error-related negativity (ERN), the waveforms were averaged across congruent and incongruent trials, no-incentive and reward trials, and $\mathrm{Fz}, \mathrm{FCz}$, and $\mathrm{Cz}$. (B) Mean amplitude scores for the ERN and the correct-response negativity (CRN) averaged across $\mathrm{Fz}, \mathrm{FCz}$, and $\mathrm{Cz}$ for the MDD and control participants. Error bars represent standard errors of the means. 
A

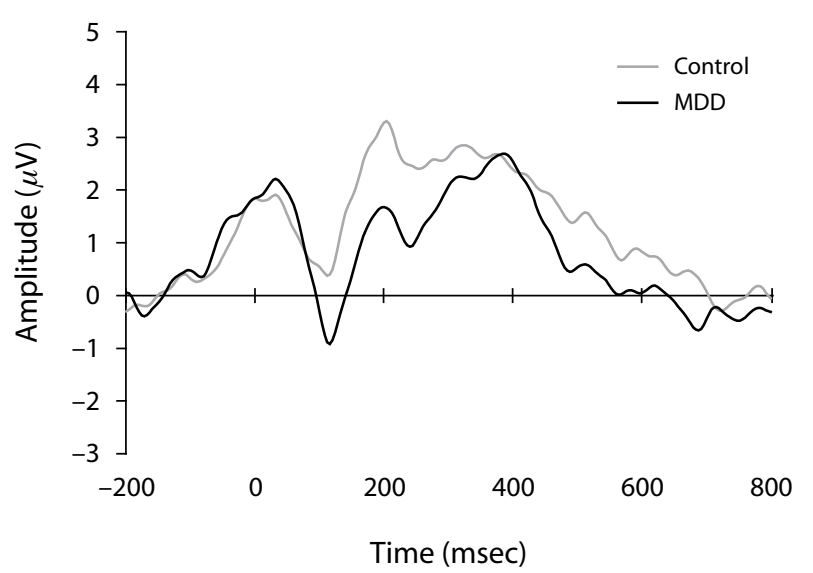

B

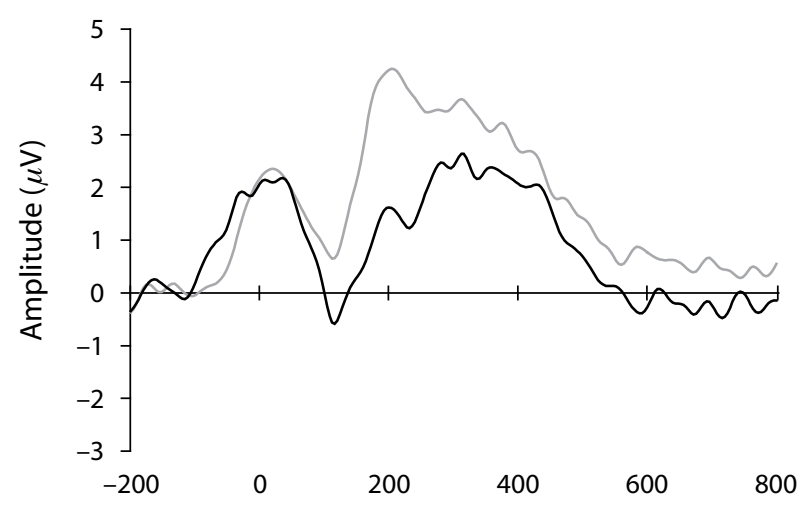

Figure 2. Response-locked waveforms for major depressive disorder (MDD; $n=13$ ) and control $(n=17)$ participants for (A) noincentive and $(B)$ reward trials. The waveform was averaged across congruent and incongruent trials and across $\mathrm{CPz}$ and $\mathrm{Pz}$ to highlight the error positivity $(\mathrm{Pe})$.

tests revealed that this interaction was driven by increased ERN amplitudes for the MDD $(-4.65 \pm 2.86 \mu \mathrm{V})$ relative to the control $(-2.94 \pm 1.77 \mu \mathrm{V} ; p<.053)$ participants (Figure 1). Importantly, no significant group differences emerged when considering CRN amplitude (MDD, $-1.04 \pm 0.13$; control, $-0.76 \pm 0.09 ; p>.47$ ). Additionally, both groups displayed more negative amplitudes for the ERN than for the CRN ( $p$ s $<.001)$. No other effects involving either group or condition approached significance (all $F_{\mathrm{s}}<2.47, p \mathrm{~s}>.12$ ).

Contrary to the findings of prior studies (Ganushchak \& Schiller, 2008; Gehring \& Willoughby, 2002; Hajcak, Moser, et al., 2005), the ERN did not differ between the reward and no-incentive conditions for control participants $(p=.17)$. Similar null findings emerged for MDD participants $(p=.89)$.

A

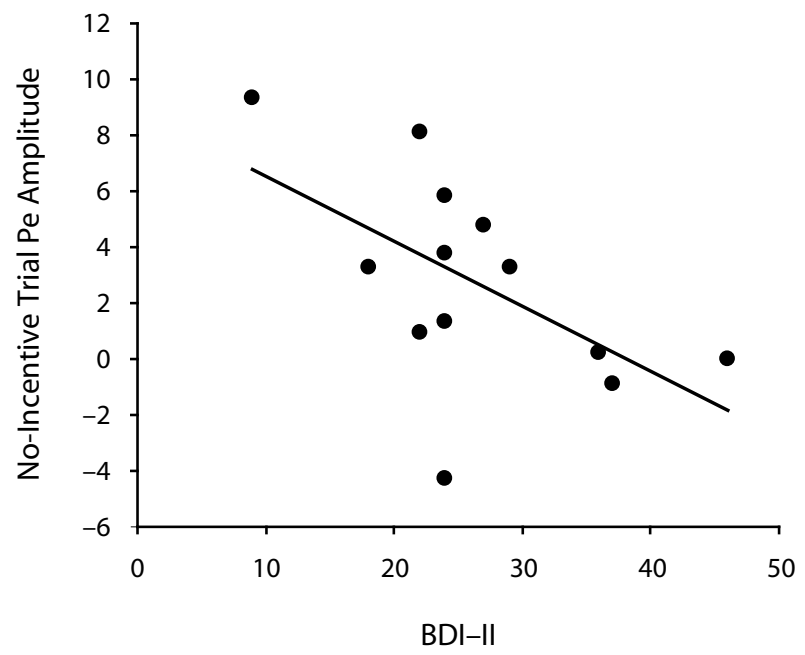

Pe

Pe analysis included no-incentive versus reward trials collapsed across incongruent and congruent trials. Significant effects emerging from the group $\times$ condition $\times$ site ANOVA on Pe amplitudes are summarized in Tables 3 and 4 . There was a main effect of site $[F(1,28)=17.85$, $p<.001 ; \eta_{\mathrm{p}}^{2}=.39$ ], due to increased Pe amplitude for $\mathrm{CPz}$ $(4.08 \pm 3.84 \mu \mathrm{V})$ relative to $\mathrm{Pz}(2.31 \pm 3.92 \mu \mathrm{V})$. Additionally, and of primary importance to our hypotheses, the group $\times$ condition interaction was significant $[F(1,28)=$ $\left.4.52, p<.043 ; \eta_{\mathrm{p}}^{2}=.14\right]$. Follow-up tests indicated that, although groups did not differ in their Pe amplitudes during no-incentive trials (controls, $3.23 \pm 3.47 \mu \mathrm{V}$; MDD, $2.75 \pm 3.76 \mu \mathrm{V} ; p=.42$; Figure $2 \mathrm{~A}$ ), controls had significantly larger Pe amplitudes than did MDD participants for the reward condition $(4.37 \pm 4.47 \mu \mathrm{V}$ vs. $2.07 \pm 3.65 \mu \mathrm{V}$;

B

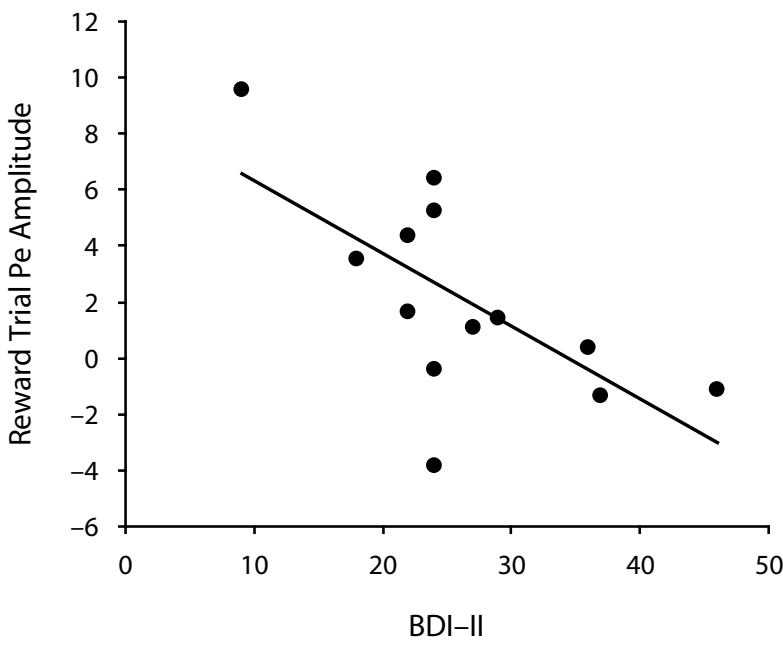

Figure 3. Scatterplots and Pearson correlations between error-positivity (Pe) amplitudes and Beck Depression Inventory-II (BDI-II) scores for (A) no-incentive (B) and reward trials for MDD participants only $(n=13)$. 
A

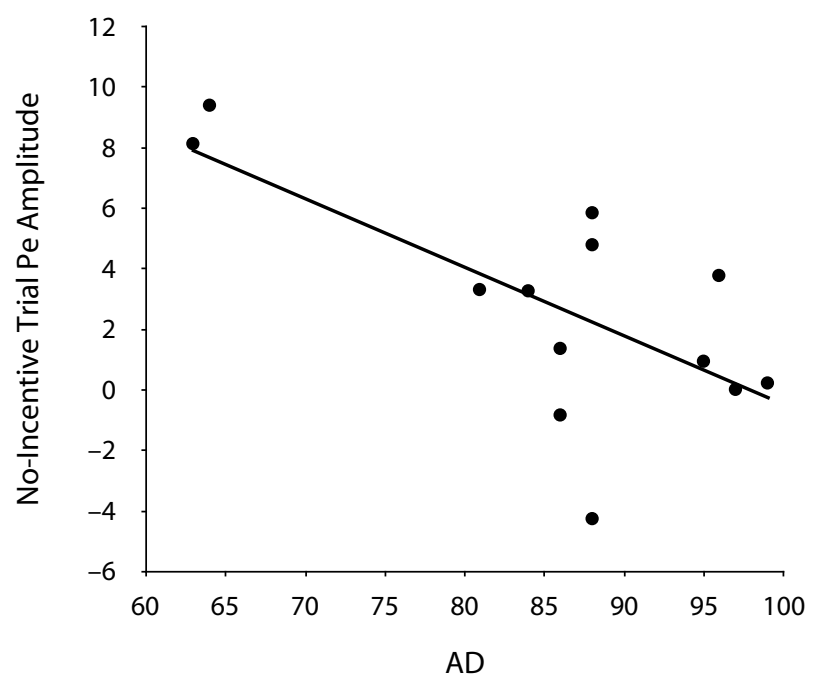

C

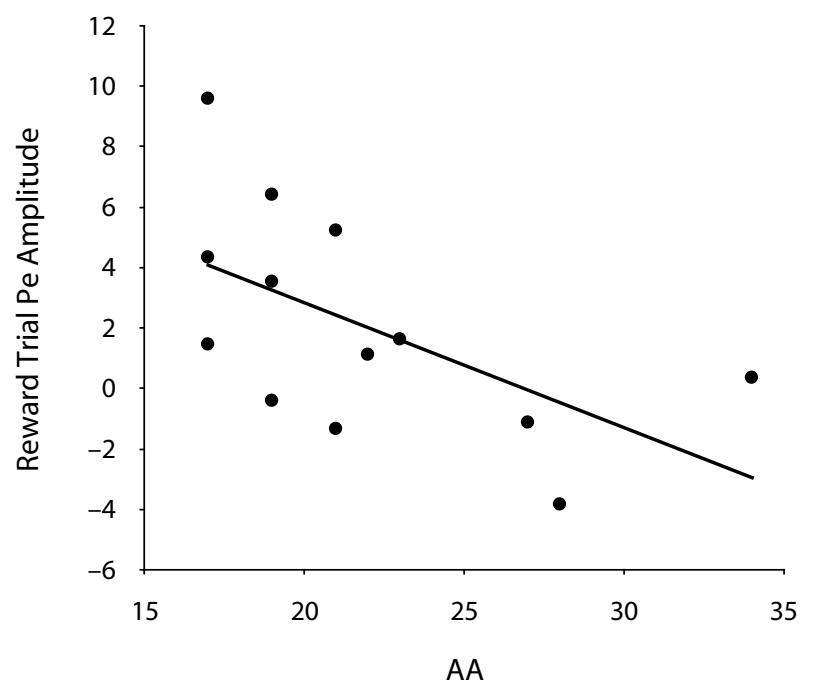

B

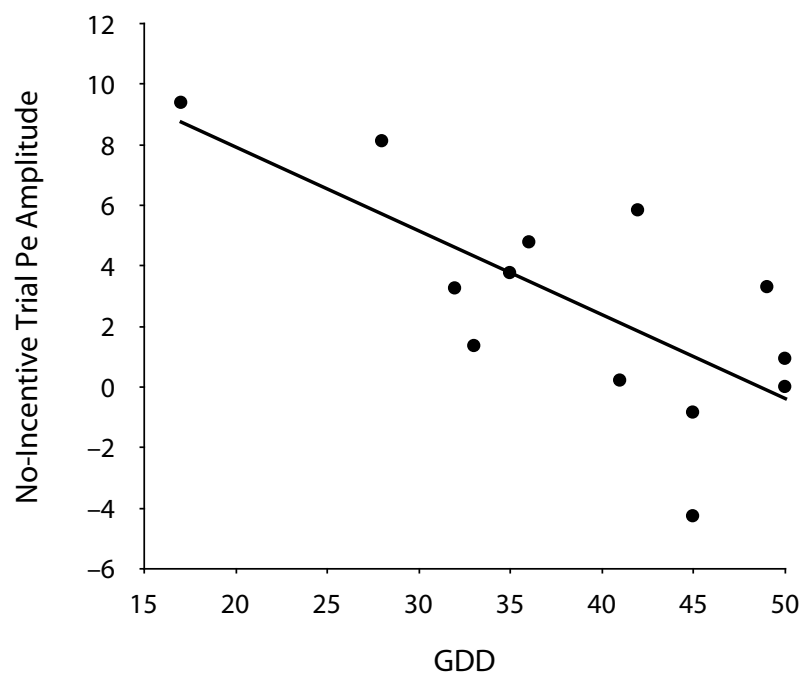

D

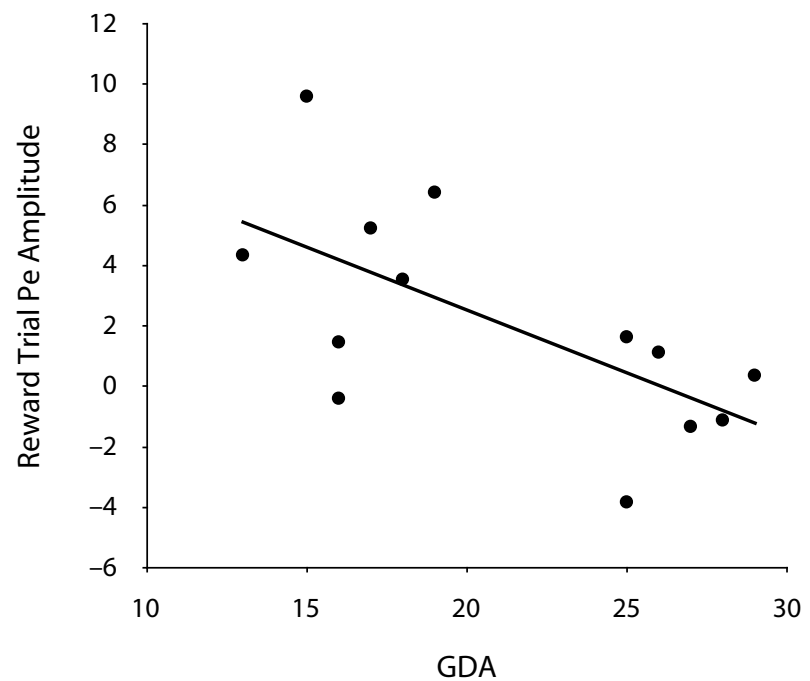

Figure 4. Scatterplots and Pearson correlations between error-positivity (Pe) amplitudes and Mood and Anxiety Symptom Questionnaire subscales for major depressive disorder participants only $(n=13)$ : $(A)$ anhedonia depression symptoms (AD), (B) general distress-depressive symptoms (GDD), (C) anxious arousal symptoms (AA), (D) general distress-anxious symptoms (GDA). For panels A and $B, P e$ amplitudes were derived from no-incentive trials. For panels $C$ and $D, P e$ amplitudes were derived from reward trials.

$p<.004$; Figure 2B). In addition, controls displayed a trend for increased Pe for reward relative to no-incentive trials $(p<.07)$, whereas MDD participants did not display any Pe modulation across conditions $(p=.27)$.

\section{Correlation Between Self-Report and ERN/Pe Amplitudes}

Among MDD participants, no significant correlations emerged between ERN amplitudes (reward or no-incentive trials) and BDI scores (all $r \mathrm{~s}<-.35$, all $p \mathrm{~s}>.23$ ) or any of the MASQ subscales (all $r \mathrm{~s}<-.45$, all $p \mathrm{~s}>.12$ ). When considering the $\mathrm{Pe}, \mathrm{BDI}$ scores were inversely related to $\mathrm{Pe}$ amplitudes for both the no-incentive $(r=-.57, p<.041$;
Figure 3A) and reward $(r=-.66, p<.015$; Figure 3B) trials. For the MASQ subscales, the Pe amplitudes during no-incentive trials were significantly correlated with AD $(r=-.68, p<.006$; Figure 4A) and GDD $(r=-.72$, $p<.006$; Figure 4B) scores, but not with GDA $(r=-.37$, $p=.21)$ or AA $(r=-.47, p=.10)$ scores. The correlation involving $\mathrm{AD}$ scores was driven by 2 MDD participants with low anhedonic symptoms, however, and should therefore be interpreted cautiously. Conversely, for reward trials, Pe amplitudes were significantly correlated with $\mathrm{AA}(r=-.58, p<.040$; Figure $4 \mathrm{C})$ and GDA $(r=-.65$, $p<.020$; Figure 4D) scores, but not with $\operatorname{GDD}(r=-.47$, $p=.10)$ or $\mathrm{AD}(r=-.32, p=.27)$ scores. 


\section{DISCUSSION}

The overarching goal of the present study was to examine action monitoring - specifically error processingin unmedicated and moderately depressed participants as a function of task-relevant incentives and clinical profile. Three main findings emerged. First, MDD participants were characterized by potentiated ERN responses, irrespective of task incentives, highlighting generally exaggerated reactivity in early (automatic) stages of error processing. Second, when monetary rewards were available, MDD participants exhibited reduced Pe amplitudes, highlighting blunted responses at later stages assumed to index conscious error awareness and subjective affective evaluative responses (Falkenstein et al., 2000). Third, whereas ERN amplitudes were not modulated by depression severity or clinical symptoms, significant negative correlations between BDI/MASQ scores and Pe amplitudes emerged, indicating that the most severely depressed participants were characterized by the lowest Pe responses.

The present ERN findings replicate prior reports of potentiated ERN responses in MDD, particularly in samples tested under no-incentive conditions (Alexopoulos et al., 2007; Holmes \& Pizzagalli, 2008; but see Compton et al., 2008; Ruchsow et al., 2006; Ruchsow et al., 2004; Schrijvers et al., 2008; Schrijvers, de Bruijn, et al., 2009). Two additional findings extend these prior studies, however. First, ERN differences were not modulated by task incentives (the significant group $\times$ response interaction was not qualified by the group $\times$ response $\times$ condition triple interaction), highlighting global ERN potentiation in moderately depressed and unmedicated MDD participants. Second, groups did not differ in their CRN amplitudes, suggesting that abnormal action monitoring is specific to error responses at early stages of processing. Of note, a recent study found that the ERN might prime defensive motivational responses (Hajcak \& Foti, 2008). Taken together, these findings indicate that depression is characterized by exaggerated automatic responses to perceived failures, which might foster the emergence or maintenance of negative processing biases (Chiu \& Deldin, 2007; Holmes \& Pizzagalli, 2008). In addition, replicating prior findings (Compton et al., 2008; Holmes \& Pizzagalli, 2008; Schrijvers et al., 2008; but see Chiu $\&$ Deldin, 2007; Schrijvers, de Bruijn, et al., 2009), the lack of correlation between ERN amplitude and clinical symptoms is consistent with recent proposals that the ERN may be associated with "stable characteristics related to internalizing disorders" (Olvet \& Hajcak, 2008, p. 1349). Reports of increased ERN amplitudes in asymptomatic individuals carrying allelic variations within the promoter region of the serotonin transporter (Althaus et al., 2009; Fallgatter et al., 2004), previously linked to increased vulnerability to depression (Caspi et al., 2003), are also consistent with this argument. Due to the relatively limited sample size investigated here, further studies are needed in order to further evaluate putative relations between ERN amplitude and clinical profile.
As compared with controls, MDD participants exhibited significantly lower Pe amplitudes following errors committed in reward trials, although no group differences were observed in the no-incentive condition. Moreover, among the MDD participants, significant negative correlations between depression severity and Pe amplitudes emerged, consistent with prior reports of reduced Pe in severely depressed individuals (Alexopoulos et al., 2007; Schrijvers et al., 2008). The present finding of decreased $\mathrm{Pe}$ in the reward-but not in the no-incentive-condition is intriguing, particularly when considering recent data in healthy controls indicating that heightened Pe amplitudes occur when errors are motivationally salient (Overbeek et al., 2005) and are associated with increased relevance to task goals (Boksem et al., 2006; Heldmann, Rüsseler, $\&$ Münte, 2005). In MDD participants, reduced Pe amplitudes are evident in severely depressed samples, a finding hypothesized to stem from increased task disengagement due to apathy/anhedonia (Schrijvers et al., 2008; Schrijvers, de Bruijn, et al., 2009). Accordingly, the present data suggest that decreased Pe amplitudes become apparent in moderately depressed individuals when monetary incentives are made available and the relationship between anhedonia and action monitoring is more explicitly probed. The correlations between Pe amplitude and (1) anhedonic symptoms and (2) negative affect among MDD participants are in line with recent data indicating that the Pe is potentiated in individuals with high reward sensitivity (e.g., Boksem et al., 2008; Boksem et al., 2006) and reduced in those with high negative affect (e.g., Hajcak, McDonald, \& Simons, 2004). However, it is important to note that anhedonic symptoms were associated with reduced Pe amplitudes in no-incentive trials (and the correlation was driven mostly by 2 MDD participants), whereas increased anxious arousal was unexpectedly linked to reduced Pe amplitudes in reward trials. The reasons for this dissociation are not clear, and future research will be necessary to determine how individual differences in selfreported anhedonia and anxiety interact with the actionmonitoring system.

Generally, increased ERN amplitudes and reduced Pe to errors occurring within reward contingencies are consistent with a failure within the MDD group to appropriately react to mistakes in performance and recruit additional resources when motivationally required. Prior research has indicated that MDD participants are characterized by reduced functional coupling between DLPFC and ACC regions immediately following error commission (Holmes \& Pizzagalli, 2008) and the presentation of personally relevant negative stimuli (Siegle et al., 2007). These findings are of interest, particularly when considering the role of ACC and DLPFC regions in cognitive control (Botvinick, Cohen, \& Carter, 2004) and reward responsiveness (Kumar et al., 2008). This literature suggests that the present data and earlier reports of abnormal reactions to errors and negative feedback in depression (Beats et al., 1996; Elliott et al., 1996; Steffens, Wagner, Levy, Horn, \& Krishnan, 2001) could emerge as a result of exaggerated paralimbic activation and/or of a failure to recruit PFC-based cognitive control, possibly due to abnormal frontocingulate connectivity. 
Findings from this study should be interpreted in the context of several limitations. First, although participants exhibited slower RTs for incongruent than for congruent trials and for no-incentive than for reward trials, no group differences were observed in the behavioral data. This allows for the ERP data to be interpreted without the possible confounds associated with differences in task performance, but it is inconsistent with prior findings of a relationship between less adaptive incongruence (Stroop) effects and depression (Holmes \& Pizzagalli, 2007). Second, there was an insufficient number of artifact-free ERP segments for performing analyses that concurrently examined condition (no incentive, reward), trial type (incongruent, congruent), and response (correct, incorrect) that would have allowed us to fully dissociate error processing and conflict monitoring. Finally, clinical symptomatology was assessed exclusively through self-report measures rather than clinician-rated measures. We cannot exclude the possibility that self-reporting biases might have partially confounded some of the correlations described in the present analyses. Given these limitations, the present findings await replications in larger samples before conclusive interpretations about the possible differential impact of task-relevant incentives on the electrophysiological correlates of action monitoring in MDD can be made.

These limitations notwithstanding, the present findings are consistent with the hypotheses that MDD is associated with a heightened reactivity to error commission and with a failure to increase activity in the presence of task-relevant incentives. The inclusion of trial-to-trial reward manipulations uncovered action-monitoring dysregulation in the present unmedicated and moderately depressed sample, with evidence of generally potentiated early (automatic) abnormal reaction to errors (ERN) but blunted responses under reward conditions at later stages associated with motivational significance of errors (Pe). Given that $a b-$ normal executive function and ERN/Pe amplitudes have been shown to predict symptom remission and antidepressant treatment response in elderly depressed patients (Alexopoulos et al., 2007; Kalayam \& Alexopoulos, 2003; Sneed et al., 2007), additional research examining the relationship between ERP correlates of action monitoring, depression, and task-relevant incentives is warranted.

\section{AUTHOR NOTE}

This research was supported by a Sackler Scholar in Psychobiology Research Grant (to A.J.H.) and NIH Grants 1 F31MH078346 (to A.J.H.) and R01MH68376 (to D.A.P.). The authors thank Ryan Bogdan and Daniel G. Dillon for their contributions and assistance with various aspects of this research. D.A.P. has received research support from GlaxoSmithKline and Merck \& Co., Inc. and consulting fees from ANT North America, Inc. (Advanced Neuro Technology) for studies unrelated to this project. A.J.H. reports no competing interests. Correspondence concerning this article should be addressed to D. A. Pizzagalli, Department of Psychology, Harvard University, 33 Kirkland St., Cambridge, MA 02138 (e-mail: dap@wjh.harvard.edu).

\section{REFERENCES}

Alexopoulos, G. S., Kiosses, D. N., Heo, M., Murphy, C. F., ShanMUgham, B., \& GUNning-Dixon, F. (2005). Executive dysfunction and the course of geriatric depression. Biological Psychiatry, 58, 204-210.
Alexopoulos, G. S., Murphy, C. F., Gunning-Dixon, F. M., KalAYAM, B., Katz, R., Kanellopoulos, D., Et AL. (2007). Eventrelated potentials in an emotional go/no-go task and remission of geriatric depression. NeuroReport, 18, 217-221.

Althaus, M., Groen, Y., Wijers, A. A., Mulder, L. J., Minderaa, R. B., Kema, I. P., ET AL. (2009). Differential effects of 5-HTTLPR and DRD2/ANKK1 polymorphisms on electrocortical measures of error and feedback processing in children. Clinical Neurophysiology, 120, 93-107.

American Psychiatric Association (1994). Diagnostic and statistical manual of mental disorders (4th ed.). Washington, DC: Author.

Beats, B. C., Sahakian, B. J., \& Levy, R. (1996). Cognitive performance in tests sensitive to frontal lobe dysfunction in the elderly depressed. Psychological Medicine, 26, 591-603.

Beck, A. T., Steer, R. A., \& Brown, G. K. (1996). Beck Depression Inventory manual (2nd ed.). San Antonio, TX: Psychological Corporation.

Boksem, M. A. S., Tops, M., Kostermans, E., \& De Cremer, D. (2008). Sensitivity to punishment and reward omission: Evidence from errorrelated ERP components. Biological Psychology, 79, 185-192.

Boksem, M. A. S., Tops, M., Wester, A. E., Meijman, T. F., \& Lorist, M. M. (2006). Error-related ERP components and individual differences in punishment and reward sensitivity. Brain Research, 1101, 92-101.

Botvinick, M. M., Cohen, J. D., \& Carter, C. S. (2004). Conflict monitoring and anterior cingulate cortex: An update. Trends in Cognitive Sciences, 8, 539-546.

Caspi, A., Sugden, K., Moffitt, T. E., Taylor, A., Craig, I. W., HarRINGTON, H., ET AL. (2003). Influence of life stress on depression: Moderation by a polymorphism in the 5-HTT gene. Science, 301, 386-389.

Chapman, L. J., \& Chapman, J. P. (1987). The measurement of handedness. Brain \& Cognition, 6, 175-183.

Chiu, P. H., \& Deldin, P. J. (2007). Neural evidence for enhanced error detection in major depressive disorder. American Journal of Psychiatry, 164, 608-616.

Compton, R. J., Lin, M., Vargas, G., Carp, J., Fineman, S. L., \& QuANDT, L. C. (2008). Error detection and posterror behavior in depressed undergraduates. Emotion, 8, 58-67.

Elliott, R., Sahakian, B. J., McKay, A. P., Herrod, J. J., Robbins, T. W., \& PAYKeL, E. S. (1996). Neuropsychological impairments in unipolar depression: The influence of perceived failure on subsequent performance. Psychological Medicine, 26, 975-989.

Falkenstein, M., Hohnsbein, J., Hoormann, J., \& Blanke, L. (1991). Effects of crossmodal divided attention on late ERP components: II. Error processing in choice reaction tasks. Electroencephalography \& Clinical Neurophysiology, 78, 447-455.

Falkenstein, M., Hoormann, J., Christ, S., \& Hohnsbein, J. (2000). ERP components on reaction errors and their functional significance: A tutorial. Biological Psychology, 51, 87-107.

Fallgatter, A. J., Herrmann, M. J., Roemmler, J., Ehlis, A.-C., WAGENER, A., HeIDrich, A., ET AL. (2004). Allelic variation of serotonin transporter function modulates the brain electrical response for error processing. Neuropsychopharmacology, 29, 1506-1511.

First, M. B., Spitzer, R. L., Gibbon, M., \& Williams, J. B. W. (2007). Structured clinical interview for DSM-IV-TR axis I disorders (Patient ed.) New York: Biometrics Research, New York State Psychiatric Institute.

Forbes, E. E., Christopher, M. J., Siegle, G. J., Ladouceur, C. D., Ryan, N. D., CARTER, C. S., ET AL. (2006). Reward-related decisionmaking in pediatric major depressive disorder: An fMRI study. Journal of Child Psychology \& Psychiatry, 47, 1031-1040.

GANUSHCHAK, L. Y., \& SCHILlER, N. O. (2008). Motivation and semantic context affect brain error-monitoring activity: An event-related brain potentials study. NeuroImage, 39, 395-405.

Gehring, W. J., Goss, B., Coles, M. G. H., Meyer, D. E., \& DonCHIN, E. (1993). A neural system for error detection and compensation. Psychological Science, 4, 385-390.

Gehring, W. J., \& Willoughby, A. R. (2002). The medial frontal cortex and the rapid processing of monetary gains and losses. Science, 295, 2279-2282.

HAJCAK, G., \& Foti, D. (2008). Errors are aversive: Defensive motivation and the error-related negativity. Psychological Science, 19, 103-108. 
Hajcak, G., Holroyd, C. B., Moser, J. S., \& Simons, R. F. (2005). Brain potentials associated with expected and unexpected good and bad outcomes. Psychophysiology, 42, 161-170.

HaJCaK, G., McDonald, N., \& Simons, R. F. (2003). To err is autonomic: Error-related brain potentials, ANS activity, and post-error compensatory behavior. Psychophysiology, 40, 895-903.

HaJCAK, G., McDonald, N., \& Simons, R. F. (2004). Error-related psychophysiology and negative affect. Brain \& Cognition, 56, 189-197.

Hajcak, G., Moser, J. S., Yeung, N., \& Simons, R. F. (2005). On the ERN and the significance of errors. Psychophysiology, 42, 151-160.

Heldmann, M., RÜsseler, J., \& MünTe, T. F. (2005). Event-related potentials in a decision-making task with delayed and immediate reward conditions. Journal of Psychophysiology, 19, 270-274.

Holmes, A. J., \& Pizzagalli, D. A. (2007). Task feedback effects on conflict monitoring and executive control: Relationship to subclinical measures of depression. Emotion, 7, 68-76.

Holmes, A. J., \& Pizzagalli, D. A. (2008). Spatiotemporal dynamics of error processing dysfunctions in major depressive disorder. $\mathrm{Ar}$ chives of General Psychiatry, 65, 179-188.

Holroyd, C. B., \& Coles, M. G. H. (2002). The neural basis of human error processing: Reinforcement learning, dopamine, and the errorrelated negativity. Psychological Review, 109, 679-709.

Jung, T.-P., Makeig, S., Westerfield, M., Townsend, J., Courchesne, E., \& SEJNOWski, T. J. (2000). Removal of eye activity artifacts from visual event-related potentials in normal and clinical subjects. Clinical Neurophysiology, 111, 1745-1758.

Kalayam, B., \& Alexopoulos, G. S. (2003). A preliminary study of left frontal region error negativity and symptom improvement in geriatric depression. American Journal of Psychiatry, 160, 2054-2056.

Kumar, P., Waiter, G., Ahearn, T., Milders, M., Reid, I., \& Steele, J. D. (2008). Abnormal temporal difference reward-learning signals in major depression. Brain, 131, 2084-2093.

LEUTHOLD, H., \& SOMMER, W. (1999). ERP correlates of error processing in spatial S-R compatibility tasks. Clinical Neurophysiology, 110, 342-357.

Luu, P., Collins, P., \& Tucker, D. M. (2000). Mood, personality, and self-monitoring: Negative affect and emotionality in relation to frontal lobe mechanisms of error monitoring. Journal of Experimental Psychology: General, 129, 43-60.

Luu, P., Tucker, D. M., Derryberry, D., Reed, M., \& Poulsen, C. (2003). Electrophysiological responses to errors and feedback in the process of action regulation. Psychological Science, 14, 47-53.

Nieuwenhuis, S., Ridderinkhof, K. R., Blom, J., Band, G. P. H., \& KoK, A. (2001). Error-related brain potentials are differentially related to awareness of response errors: Evidence from an antisaccade task. Psychophysiology, 38, 752-760.

OLVET, D. M., \& HAJCAK, G. (2008). The error-related negativity (ERN) and psychopathology: Toward an endophenotype. Clinical Psychology Review, 28, 1343-1354.

Overbeek, T. J. M., Nieuwenhuis, S., \& Ridderinkhof, K. R. (2005). Dissociable components of error processing: On the functional significance of the Pe vis-à-vis the ERN/Ne. Journal of Psychophysiology, 19, 319-329.

Pizzagalli, D. A., Dillon, D. G., Bogdan, R., \& Holmes, A. J. (in press). Reward and punishment processing in the human brain: Clues from affective neuroscience and implications for depression research. In O. Vartanian \& D. R. Mandel (Eds.), Neuroscience of decision making. New York: Psychology Press.

Pizzagalli, D. A., Holmes, A. J., Dillon, D. G., Goetz, E. L., Birk, J. L., Bogdan, R., ET AL. (2009). Reduced caudate and nucleus ac- cumbens response to rewards in unmedicated individuals with major depressive disorder. American Journal of Psychiatry, 166, 702-710.

Pizzagalli, D. A., Iosifescu, D., Hallett, L. A., Ratner, K. G., \& FAVA, M. (2009). Reduced hedonic capacity in major depressive disorder: Evidence from a probabilistic reward task. Journal of Psychiatric Research, 43, 76-87.

Ruchsow, M., Herrnberger, B., Beschoner, P., Grön, G., SPITZER, M., \& KiefER, M. (2006). Error processing in major depressive disorder: Evidence from event-related potentials. Journal of Psychiatric Research, 40, 37-46.

Ruchsow, M., Herrnberger, B., Wiesend, C., Grön, G., Spitzer, M., $\&$ KIEFER, M. (2004). The effect of erroneous responses on response monitoring in patients with major depressive disorder: A study with event-related potentials. Psychophysiology, 41, 833-840.

Schrijvers, D., De Bruijn, E. R. A., Maas, Y. J., De Grave, C., Sabbe, B. G. C., \& HulstiJn, W. (2008). Action monitoring in major depressive disorder with psychomotor retardation. Cortex, 44, 569-579.

Schrijvers, D., De Bruijn, E. R. A., MaAs, Y. J., Vancoillie, P., HulStiJn, W., \& SABBE, B. G. C. (2009). Action monitoring and depressive symptom reduction in major depressive disorder. International Journal of Psychophysiology, 71, 218-224.

Schrijvers, D., Maas, Y. J., Pier, M. P., Madani, Y., Hulstijn, W., \& SABbe, B. G. C. (2009). Psychomotor changes in major depressive disorder during sertraline treatment. Neuropsychobiology, 59, $34-42$.

Shalgi, S., Barkan, I., \& Deouell, L. Y. (2009). On the positive side of error processing: Error-awareness positivity revisited. European Journal of Neuroscience, 29, 1522-1532.

Siegle, G. J., Thompson, W., Carter, C. S., Steinhauer, S. R., \& Thase, M. E. (2007). Increased amygdala and decreased dorsolateral prefrontal BOLD responses in unipolar depression: Related and independent features. Biological Psychiatry, 61, 198-209.

Sneed, J. R., Roose, S. P., Keilp, J. G., Krishnan, K. R. R., AlexoPOULOS, G. S., \& SACKEIM, H. A. (2007). Response inhibition predicts poor antidepressant treatment response in very old depressed patients. American Journal of Geriatric Psychiatry, 15, 553-563.

Steffens, D. C., Wagner, H. R., Levy, R. M., Horn, K. A., \& KRISHNAN, K. R. R. (2001). Performance feedback deficit in geriatric depression. Biological Psychiatry, 50, 358-363.

Tucker, D. M., Luu, P., Frishroff, G., Quiring, J., \& Poulsen, C. (2003). Frontolimbic response to negative feedback in clinical depression. Journal of Abnormal Psychology, 112, 667-678.

Ullsperger, M., \& von Cramon, D. Y. (2001). Subprocesses of performance monitoring: A dissociation of error processing and response competition revealed by event-related fMRI and ERPs. NeuroImage, 14, 1387-1401.

Ullsperger, M., \& von Cramon, D. Y. (2006). How does error correction differ from error signaling? An event-related potential study. Brain Research, 1105, 102-109.

VAN VeEN, V., \& CARTER, C. S. (2002). The timing of action-monitoring processes in the anterior cingulate cortex. Journal of Cognitive Neuroscience, 14, 593-602.

Watson, D., Weber, K., Assenheimer, J. S., Clark, L. A., Strauss, M. E., \& McCormick, R. A. (1995). Testing a tripartite model: I. Evaluating the convergent and discriminant validity of anxiety and depression symptom scales. Journal of Abnormal Psychology, 104, 3-14.

(Manuscript received May 2, 2009; revision accepted for publication September 6, 2009.) 